\title{
Quantum Weak Energy Inequalities for the Dirac field in Flat Spacetime
}

\author{
C.J. Fewster * and B. Mistry \\ Department of Mathematics, University of York, Heslington, York, YO10 5DD, UK
}

(Dated: 15 September 2003)

\begin{abstract}
Quantum Weak Energy Inequalities (QWEIs) have been established for a variety of quantum field theories in both flat and curved spacetimes. Dirac fields are known (by a result of Fewster and Verch) to satisfy QWEIs under very general circumstances. However this result does not provide an explicit formula for the QWEI bound, so its magnitude has not previously been determined. In this paper we present a new and explicit QWEI bound for Dirac fields of mass $m \geqslant 0$ in fourdimensional Minkowski space. We follow the methods employed by Fewster and Eveson for the scalar field, modified to take account of anticommutation relations. A key ingredient is an identity for Fourier transforms established by Fewster and Verch. We also compare our QWEI with those previously obtained for scalar and spin-1 fields.
\end{abstract}

PACS numbers: 03.70.+k, 11.10.Ef

\section{INTRODUCTION}

It has been known for a long time that the energy density of a quantum field may assume negative values [1]. If the magnitude and the duration of such negative energy densities were unconstrained various exotic phenomema might be possible, ranging from the manufacture of macroscopic traversable wormholes [2] to large-scale violations of the second law of thermodynamics [3].

However, quantum field theory itself contains mechanisms to limit the extent of negative energy densities: certain weighted averages of the stress-energy tensor obey state-independent lower bounds known as quantum weak energy inequalities (QWEI) [or simply quantum inequalities (QI)] which severely limit exotic phenomena such as those described above.

Bounds of this type have been obtained by various means [4, 5, 6, 7, 8, 9, 10, 11, 12, 13, 14] for the scalar, electromagnetic and Proca fields, in both flat and curved spacetimes, leading to results of great generality. Taking four-dimensional Minkowski space as a specific example, and averaging the energy density along an inertial worldline, the QWEI satisfied by these three theories may be written in the form [8, 9, 11, 12 ]

$$
\int d t\left\langle: T_{00}:\right\rangle_{\psi}\left(t, \boldsymbol{x}_{\mathbf{0}}\right) g(t)^{2} \geqslant-\frac{\mathcal{S}}{16 \pi^{3}} \int_{m}^{\infty} d u|\widehat{g}(u)|^{2} u^{4} Q_{3}(u / m)
$$

for any smooth, real-valued $g$ vanishing outside a compact region, where $m$ is the particle's mass, $\mathcal{S}$ denotes its helicity ( $\mathcal{S}=1$ for scalars, 2 for photons and 3 for massive spin-1 particles), while

$$
Q_{3}(x)=\left(1-\frac{1}{x^{2}}\right)^{1 / 2}\left(1-\frac{1}{2 x^{2}}\right)-\frac{1}{2 x^{4}} \ln \left(x+\sqrt{x^{2}-1}\right)
$$

and is replaced by $\lim _{x \rightarrow \infty} Q_{3}(x)=1$ in the massless case. (In curved spacetimes the QWEIs of these theories are not simply related by an overall factor.)

In the spin- $\frac{1}{2}$ case only two arguments are known. One, due to Vollick [15] (based on earlier work of Flanagan [6]), exploits the conformal properties of massless Dirac fields in two-dimensional spacetimes to obtain a sharp (and explicit) QWEI for an arbitrary sampling function. The other, due to Fewster and Verch [16], establishes the existence of QEWIs for Dirac and Majorana fields of arbitrary mass in general four-dimensional globally hyperbolic spacetimes by drawing on techniques from microlocal analysis. However, this approach did not lead to an explicit formula for the QWEI bound, so its magnitude - even in four-dimensional Minkowski space - has not previously been determined [20]. (Specific classes of Dirac states with local negative energy densities have been studied [17, 18, and shown, in the massless limit, to satisfy the scalar field QWEI obtained in 5]; of course, this does not amount to a proof of the QWEIs.) Thus, for example, it has not previously been clear whether or not it is more or less easy to support a traversable wormhole using Dirac fields, rather than with the scalar or electromagnetic fields.

*Electronic address: cjf3@york.ac.uk 
In this paper, we present a new QWEI derivation for Dirac fields, which combines ideas taken from 16 with an approach first developed for the scalar field in [8, 9]. The argument is straightforward, involving the use of anticommutation relations and an identity for Fourier transforms, and yields an explicit, closed-form, bound. In four-dimensional Minkowski space this bound is

$$
\int d t\left\langle: T_{00}:\right\rangle_{\psi}\left(t, \boldsymbol{x}_{\mathbf{0}}\right) g(t)^{2} \geqslant-\frac{1}{12 \pi^{3}} \int_{m}^{\infty} d u|\widehat{g}(u)|^{2} u^{4} Q_{3}^{D}(u / m),
$$

where

$$
Q_{3}^{D}(x)=4\left(1-\frac{1}{x^{2}}\right)^{3 / 2}-3 Q_{3}(x)
$$

is replaced by $\lim _{x \rightarrow \infty} Q_{3}(x)=1$ in the massless case. Although we have restricted ourselves, for simplicity, to Minkowski space, our technique certainly extends to static spacetimes and may even suggest a new approach in the general globally hyperbolic case. It is hoped to return to these issues elsewhere.

Conventions: We take the metric to have signature $(+---)$ and adopt units in which $\hbar=c=1$. The Fourier transform $\widehat{f}$ of a function $f$ on $\mathbb{R}$ is defined by

$$
\widehat{f}(\omega)=\int_{-\infty}^{\infty} d t f(t) e^{-i \omega t}
$$

\section{THE QWEI DERIVATION}

To establish our notation, we begin by recalling some basic facts about the Dirac fields. The Dirac equation for a fermion field $\psi$ of mass $m$ is

$$
\left(i \gamma^{\mu} \partial_{\mu}-m\right) \psi=0
$$

where the $\gamma$-matrices are given in terms of the Pauli matrices $\sigma_{i}$ by

$$
\gamma^{0}=\left(\begin{array}{cc}
1 & 0 \\
0 & -1
\end{array}\right), \quad \gamma^{i}=\left(\begin{array}{cc}
0 & \sigma_{i} \\
-\sigma_{i} & 0
\end{array}\right) \quad(i=1,2,3)
$$

and obey $\left\{\gamma^{\mu}, \gamma^{\nu}\right\}=2 \eta^{\mu \nu}$. The stress-energy tensor [19] is

$$
T_{\mu \nu}=\frac{i}{4}\left[\bar{\psi} \gamma_{\mu} \overleftrightarrow{\partial_{\nu}} \psi+\bar{\psi} \gamma_{\nu} \overleftrightarrow{\partial_{\mu}} \psi\right]
$$

where $\bar{\psi}=\psi^{\dagger} \gamma^{0}$, so the classical Dirac energy density is

$$
T_{00}=\frac{i}{2}\left[\psi^{\dagger} \dot{\psi}-\dot{\psi}^{\dagger} \psi\right]
$$

(Of course, this quantity is unbounded both from above and below, in contrast to the energy density of a classical scalar field. This is at the root of the differences between the QWEIs obeyed by the scalar and Dirac fields.) To simplify our notation, we quantise the Dirac field in a box of side $L$ and then take the continuum limit $L \rightarrow \infty$ towards the end of the argument. We emphasise that this is done purely for notational convenience. The Dirac field operator $\psi(x)$ is therefore defined in terms of creation and annihilation operators by

$$
\psi(x)=\sum_{\boldsymbol{k}} \sum_{\alpha=1,2}\left[b_{\alpha}(\boldsymbol{k}) u^{\alpha}(\boldsymbol{k}) e^{-i k \cdot x}+d_{\alpha}^{\dagger}(\boldsymbol{k}) v^{\alpha}(\boldsymbol{k}) e^{i k \cdot x}\right]
$$

where $(L / \pi) \boldsymbol{k}$ runs through $\mathbb{Z}^{3}$, and the four-vector $k$ has components $k^{a}=\left(\omega_{\boldsymbol{k}}, \boldsymbol{k}\right)$ with $\omega_{\boldsymbol{k}}=\sqrt{\|\boldsymbol{k}\|^{2}+m^{2}}$. The annihilation and creation operators satisfy the anticommutation relations

$$
\left\{b_{\alpha}(\boldsymbol{k}), b_{\alpha^{\prime}}^{\dagger}\left(\boldsymbol{k}^{\prime}\right)\right\}=\delta_{\alpha, \alpha^{\prime}} \delta_{\boldsymbol{k}, \boldsymbol{k}^{\prime}} \mathbb{1}
$$


and

$$
\left\{d_{\alpha}(\boldsymbol{k}), d_{\alpha^{\prime}}^{\dagger}\left(\boldsymbol{k}^{\prime}\right)\right\}=\delta_{\alpha, \alpha^{\prime}} \delta_{\boldsymbol{k}, \boldsymbol{k}^{\prime}} \mathbb{1},
$$

with all other anticommutators vanishing. Finally, the spinors $u^{\alpha}(\boldsymbol{k})$ and $v^{\alpha}(\boldsymbol{k})$, where $\alpha=1,2$ labels the two independent spin states, are given by

$$
u^{\alpha}(\boldsymbol{k})=\left(\begin{array}{c}
\sqrt{\frac{\omega_{\boldsymbol{k}}+m}{2 \omega_{\boldsymbol{k}} V}} \phi^{\alpha} \\
\frac{\boldsymbol{\sigma} \cdot \boldsymbol{k}}{\sqrt{2 \omega_{\boldsymbol{k}}\left(\omega_{\boldsymbol{k}}+m\right) V}} \phi^{\alpha}
\end{array}\right) \quad \text { and } \quad v^{\alpha}(\boldsymbol{k})=\left(\begin{array}{c}
\frac{\boldsymbol{\sigma} \cdot \boldsymbol{k}}{\sqrt{2 \omega_{\boldsymbol{k}}\left(\omega_{\boldsymbol{k}}+m\right) V}} \phi^{\alpha} \\
\sqrt{\frac{\omega_{\boldsymbol{k}}+m}{2 \omega_{\boldsymbol{k}} V}} \phi^{\alpha}
\end{array}\right)
$$

in which the two dimensional column vectors $\phi^{\alpha}$ are $\phi^{1 \dagger}=(1,0)$ and $\phi^{2 \dagger}=(0,1)$ and $V=L^{3}$. The normalisation has been chosen so that

$$
\sum_{\alpha}\left\|u^{\alpha}(\boldsymbol{k})\right\|^{2}=\sum_{\alpha}\left\|v^{\alpha}(\boldsymbol{k})\right\|^{2}=\frac{2}{V}
$$

Substituting the quantum field $\psi(x)$ into the classical energy density Eq. (2.4) and normal ordering according to the prescription

$$
: d_{\alpha}(\boldsymbol{k}) d_{\alpha^{\prime}}^{\dagger}\left(\boldsymbol{k}^{\prime}\right):=-d_{\alpha^{\prime}}^{\dagger}\left(\boldsymbol{k}^{\prime}\right) d_{\alpha}(\boldsymbol{k}),
$$

we obtain the normal ordered energy density at the spatial origin $(t, \mathbf{0})$ in the form

$$
\begin{aligned}
: T_{00}:(t, \mathbf{0})=\frac{1}{2} \sum_{\boldsymbol{k}, \boldsymbol{k}^{\prime}} \sum_{\alpha, \alpha^{\prime}}\left\{( \omega _ { \boldsymbol { k } } + \omega _ { \boldsymbol { k } ^ { \prime } } ) \left[b_{\alpha}^{\dagger}(\boldsymbol{k}) b_{\alpha^{\prime}}\left(\boldsymbol{k}^{\prime}\right) u^{\alpha \dagger}(\boldsymbol{k}) u^{\alpha^{\prime}}\left(\boldsymbol{k}^{\prime}\right) e^{i\left(\omega_{\boldsymbol{k}}-\omega_{\boldsymbol{k}^{\prime}}\right) t}\right.\right. \\
\left.+d_{\alpha^{\prime}}^{\dagger}\left(\boldsymbol{k}^{\prime}\right) d_{\alpha}(\boldsymbol{k}) v^{\alpha \dagger}(\boldsymbol{k}) v^{\alpha^{\prime}}\left(\boldsymbol{k}^{\prime}\right) e^{-i\left(\omega_{\boldsymbol{k}}-\omega_{\boldsymbol{k}^{\prime}}\right) t}\right] \\
\quad+\left(\omega_{\boldsymbol{k}^{\prime}}-\omega_{\boldsymbol{k}}\right)\left[d_{\alpha}(\boldsymbol{k}) b_{\alpha^{\prime}}\left(\boldsymbol{k}^{\prime}\right) v^{\alpha \dagger}(\boldsymbol{k}) u^{\alpha^{\prime}}\left(\boldsymbol{k}^{\prime}\right) e^{-i\left(\omega_{\boldsymbol{k}}+\omega_{\boldsymbol{k}^{\prime}}\right) t}\right. \\
\left.\left.\quad-b_{\alpha}^{\dagger}(\boldsymbol{k}) d_{\alpha^{\prime}}^{\dagger}\left(\boldsymbol{k}^{\prime}\right) u^{\alpha \dagger}(\boldsymbol{k}) v^{\alpha^{\prime}}\left(\boldsymbol{k}^{\prime}\right) e^{i\left(\omega_{\boldsymbol{k}}+\omega_{\boldsymbol{k}^{\prime}}\right) t}\right]\right\}
\end{aligned}
$$

We will consider weighted energy density averages of the form

$$
T_{f}=\int_{-\infty}^{\infty} d t: T_{00}:(t, \mathbf{0}) f(t)
$$

measured by a stationary observer at spacetime origin $\mathbf{0}$, where $f$ is a non-negative sampling function. Thus,

$$
\begin{aligned}
T_{f}=\frac{1}{2} & \sum_{\boldsymbol{k}, \boldsymbol{k}^{\prime}} \sum_{\alpha, \alpha^{\prime}}\left\{( \omega _ { \boldsymbol { k } } + \omega _ { \boldsymbol { k } ^ { \prime } } ) \left[b_{\alpha}^{\dagger}(\boldsymbol{k}) b_{\alpha^{\prime}}\left(\boldsymbol{k}^{\prime}\right) u^{\alpha \dagger}(\boldsymbol{k}) u^{\alpha^{\prime}}\left(\boldsymbol{k}^{\prime}\right) \widehat{f}\left(\omega_{\boldsymbol{k}^{\prime}}-\omega_{\boldsymbol{k}}\right)\right.\right. \\
& \left.+d_{\alpha^{\prime}}^{\dagger}\left(\boldsymbol{k}^{\prime}\right) d_{\alpha}(\boldsymbol{k}) v^{\alpha \dagger}(\boldsymbol{k}) v^{\alpha^{\prime}}\left(\boldsymbol{k}^{\prime}\right) \widehat{f}\left(\omega_{\boldsymbol{k}}-\omega_{\boldsymbol{k}^{\prime}}\right)\right] \\
& +\left(\omega_{\boldsymbol{k}^{\prime}}-\omega_{\boldsymbol{k}}\right)\left[d_{\alpha}(\boldsymbol{k}) b_{\alpha^{\prime}}\left(\boldsymbol{k}^{\prime}\right) v^{\alpha \dagger}(\boldsymbol{k}) u^{\alpha^{\prime}}\left(\boldsymbol{k}^{\prime}\right) \widehat{f}\left(\omega_{\boldsymbol{k}}+\omega_{\boldsymbol{k}^{\prime}}\right)\right. \\
& \left.\left.-b_{\alpha}^{\dagger}(\boldsymbol{k}) d_{\alpha^{\prime}}^{\dagger}\left(\boldsymbol{k}^{\prime}\right) u^{\alpha \dagger}(\boldsymbol{k}) v^{\alpha^{\prime}}\left(\boldsymbol{k}^{\prime}\right) \widehat{f}\left(-\omega_{\boldsymbol{k}}-\omega_{\boldsymbol{k}^{\prime}}\right)\right]\right\} .
\end{aligned}
$$

Our aim is to determine a lower bound on expectation values of $T_{f}$ in the case where $f=g^{2}$, for some real-valued, smooth, compactly supported function $g$. To this end, we first define a family $\left\{\mathcal{O}_{\mu i} \mid \mu \in \mathbb{R}, i=1,2,3,4\right\}$ of Fock space operators by

$$
\mathcal{O}_{\mu i}=\sum_{\alpha^{\prime}, \boldsymbol{k}^{\prime}}\left\{\overline{\widehat{g}\left(-\omega_{\boldsymbol{k}^{\prime}}+\mu\right)} b_{\alpha^{\prime}}\left(\boldsymbol{k}^{\prime}\right) u_{i}^{\alpha^{\prime}}\left(\boldsymbol{k}^{\prime}\right)+\overline{\widehat{g}\left(\omega_{\boldsymbol{k}^{\prime}}+\mu\right)} d_{\alpha^{\prime}}^{\dagger}\left(\boldsymbol{k}^{\prime}\right) v_{i}^{\alpha^{\prime}}\left(\boldsymbol{k}^{\prime}\right)\right\}
$$

A short calculation, using the anticommutation relations and the normalisation (2.9), shows that

$$
\begin{aligned}
\mathcal{O}_{\mu i}^{\dagger} \mathcal{O}_{\mu i}=S_{\mu} \mathbb{1}+\sum_{\alpha, \alpha^{\prime}} \sum_{\boldsymbol{k}, \boldsymbol{k}^{\prime}}\left\{\widehat{g}\left(-\omega_{\boldsymbol{k}}+\mu\right) \overline{\widehat{g}\left(-\omega_{\boldsymbol{k}^{\prime}}+\mu\right)} b_{\alpha}^{\dagger}(\boldsymbol{k}) b_{\alpha^{\prime}}\left(\boldsymbol{k}^{\prime}\right) u^{\alpha \dagger}(\boldsymbol{k}) u^{\alpha^{\prime}}\left(\boldsymbol{k}^{\prime}\right)\right. \\
-\widehat{g}\left(\omega_{\boldsymbol{k}}+\mu\right) \overline{\widehat{g}\left(\omega_{\boldsymbol{k}^{\prime}}+\mu\right)} d_{\alpha^{\prime}}^{\dagger}\left(\boldsymbol{k}^{\prime}\right) d_{\alpha}(\boldsymbol{k}) v^{\alpha \dagger}(\boldsymbol{k}) v^{\alpha^{\prime}}\left(\boldsymbol{k}^{\prime}\right) \\
+\widehat{g}\left(\omega_{\boldsymbol{k}}+\mu\right) \overline{\widehat{g}\left(-\omega_{\boldsymbol{k}^{\prime}}+\mu\right)} d_{\alpha}(\boldsymbol{k}) b_{\alpha^{\prime}}\left(\boldsymbol{k}^{\prime}\right) v^{\alpha \dagger}(\boldsymbol{k}) u^{\alpha^{\prime}}\left(\boldsymbol{k}^{\prime}\right) \\
\left.+\widehat{g}\left(-\omega_{\boldsymbol{k}}+\mu\right) \overline{\widehat{g}\left(\omega_{\boldsymbol{k}^{\prime}}+\mu\right)} b_{\alpha}^{\dagger}(\boldsymbol{k}) d_{\alpha^{\prime}}^{\dagger}\left(\boldsymbol{k}^{\prime}\right) u^{\alpha \dagger}(\boldsymbol{k}) v^{\alpha^{\prime}}\left(\boldsymbol{k}^{\prime}\right)\right\}
\end{aligned}
$$


where

$$
S_{\mu}=\frac{2}{V} \sum_{\boldsymbol{k}}\left|\widehat{g}\left(\omega_{\boldsymbol{k}}+\mu\right)\right|^{2}
$$

and we have implicitly summed over the spinor index $i$.

To relate this to our expression for $T_{f}$ we use the following Lemma, which plays an analogous role here to that played by the convolution theorem in $[8,9]$.

Lemma 1: If $f=g^{2}$ for some real-valued, smooth, compactly supported $g \in C_{0}^{\infty}(\mathbb{R})$ then

$$
\left(\omega+\omega^{\prime}\right) \widehat{f}\left(\omega-\omega^{\prime}\right)=\int_{-\infty}^{\infty} \frac{d \mu}{\pi} \mu \widehat{g}(\omega-\mu) \overline{\widehat{g}\left(\omega^{\prime}-\mu\right)} .
$$

This Lemma was also used in the QWEI derivation in [16]; for completeness we give a proof of this statement in the Appendix which differs slightly from that given in that reference. Using this Lemma with the anticommutation relations Eq. (2.6) and Eq. (2.7), we calculate

$$
\begin{aligned}
\int_{-\infty}^{\infty} d \mu \mu\left(\mathcal{O}_{\mu}^{\dagger} \mathcal{O}_{\mu}-S_{\mu} \mathbb{1}\right)=\pi \sum_{\alpha, \alpha^{\prime}} \sum_{\boldsymbol{k}, \boldsymbol{k}^{\prime}} & \left\{( \omega _ { \boldsymbol { k } } + \omega _ { \boldsymbol { k } ^ { \prime } } ) \left[b_{\alpha}^{\dagger}(\boldsymbol{k}) b_{\alpha^{\prime}}\left(\boldsymbol{k}^{\prime}\right) u^{\alpha \dagger}(\boldsymbol{k}) u^{\alpha^{\prime}}\left(\boldsymbol{k}^{\prime}\right) \widehat{f}\left(\omega_{\boldsymbol{k}^{\prime}}-\omega_{\boldsymbol{k}}\right)\right.\right. \\
& \left.+d_{\alpha^{\prime}}^{\dagger}\left(\boldsymbol{k}^{\prime}\right) d_{\alpha}(\boldsymbol{k}) v^{\alpha \dagger}(\boldsymbol{k}) v^{\alpha^{\prime}}\left(\boldsymbol{k}^{\prime}\right) \widehat{f}\left(\omega_{\boldsymbol{k}}-\omega_{\boldsymbol{k}^{\prime}}\right)\right] \\
& +\left(\omega_{\boldsymbol{k}^{\prime}}-\omega_{\boldsymbol{k}}\right)\left[d_{\alpha}(\boldsymbol{k}) b_{\alpha^{\prime}}\left(\boldsymbol{k}^{\prime}\right) v^{\alpha \dagger}(\boldsymbol{k}) u^{\alpha^{\prime}}\left(\boldsymbol{k}^{\prime}\right) \widehat{f}\left(\omega_{\boldsymbol{k}}+\omega_{\boldsymbol{k}^{\prime}}\right)\right. \\
& \left.\left.-b_{\alpha}^{\dagger}(\boldsymbol{k}) d_{\alpha^{\prime}}^{\dagger}\left(\boldsymbol{k}^{\prime}\right) u^{\alpha \dagger}(\boldsymbol{k}) v^{\alpha^{\prime}}\left(\boldsymbol{k}^{\prime}\right) \widehat{f}\left(-\omega_{\boldsymbol{k}}-\omega_{\boldsymbol{k}^{\prime}}\right)\right]\right\} .
\end{aligned}
$$

Comparing with Eq. 2.13), we conclude that

$$
T_{f}=\frac{1}{2 \pi} \int_{-\infty}^{\infty} d \mu \mu\left(\mathcal{O}_{\mu}^{\dagger} \mathcal{O}_{\mu}-S_{\mu} \mathbb{1}\right)
$$

which is similar in form to the decomposition of the scalar field energy density employed in [8]. Next we compute the anticommutator

$$
\begin{array}{r}
\left\{\mathcal{O}_{\mu i}^{\dagger}, \mathcal{O}_{\mu i}\right\}=\sum_{\alpha, \alpha^{\prime}} \sum_{\boldsymbol{k}, \boldsymbol{k}^{\prime}}\left\{\widehat{g}\left(-\omega_{\boldsymbol{k}}+\mu\right) \overline{\widehat{g}\left(-\omega_{\boldsymbol{k}^{\prime}}+\mu\right)}\left\{b_{\alpha}^{\dagger}(\boldsymbol{k}), b_{\alpha^{\prime}}\left(\boldsymbol{k}^{\prime}\right)\right\} u^{\alpha \dagger}(\boldsymbol{k}) u^{\alpha^{\prime}}\left(\boldsymbol{k}^{\prime}\right)\right. \\
\left.+\widehat{g}\left(\omega_{\boldsymbol{k}}+\mu\right) \overline{\widehat{g}\left(\omega_{\boldsymbol{k}^{\prime}}+\mu\right)}\left\{d_{\alpha}(\boldsymbol{k}), d_{\alpha^{\prime}}^{\dagger}\left(\boldsymbol{k}^{\prime}\right)\right\} v^{\alpha \dagger}(\boldsymbol{k}) v^{\alpha^{\prime}}\left(\boldsymbol{k}^{\prime}\right)\right\}
\end{array}
$$

where we have again implicitly summed over the spinor index $i$ and used the fact that anticommutators between the $b_{\alpha}(\boldsymbol{k})$ and $d_{\alpha}(\boldsymbol{k})$ vanish. Using the anticommutation relations, the above expression reduces to

$$
\begin{aligned}
\left\{\mathcal{O}_{\mu i}^{\dagger}, \mathcal{O}_{\mu i}\right\} & =\left(\sum_{\alpha, \boldsymbol{k}}\left|\widehat{g}\left(-\omega_{\boldsymbol{k}}+\mu\right)\right|^{2}\left\|u^{\alpha}(\boldsymbol{k})\right\|^{2}+\sum_{\alpha, \boldsymbol{k}}\left|\widehat{g}\left(\omega_{\boldsymbol{k}}+\mu\right)\right|^{2}\left\|v^{\alpha}(\boldsymbol{k})\right\|^{2}\right) \mathbb{1} \\
& =\left(S_{-\mu}+S_{\mu}\right) \mathbb{1},
\end{aligned}
$$

where we have also used Eq. (2.9) and the fact that $|\widehat{g}(u)|$ is even (because $g$ is real). Splitting the $\mu$-integral of Eq. (2.19) into two pieces and using Eq. (2.21) gives

$$
\begin{aligned}
T_{f} & =\frac{1}{2 \pi} \int_{0}^{\infty} d \mu \mu\left(\mathcal{O}_{\mu i}^{\dagger} \mathcal{O}_{\mu i}-S_{\mu} \mathbb{1}\right)+\frac{1}{2 \pi} \int_{-\infty}^{0} d \mu \mu\left[\left(S_{\mu}+S_{-\mu}\right) \mathbb{1}-\mathcal{O}_{\mu i} \mathcal{O}_{\mu i}^{\dagger}-S_{\mu} \mathbb{1}\right] \\
& =\frac{1}{2 \pi} \int_{0}^{\infty} d \mu \mu\left(\mathcal{O}_{\mu i}^{\dagger} \mathcal{O}_{\mu i}-S_{\mu} \mathbb{1}\right)+\frac{1}{2 \pi} \int_{-\infty}^{0} d \mu \mu\left(S_{-\mu} \mathbb{1}-\mathcal{O}_{\mu i} \mathcal{O}_{\mu i}^{\dagger}\right) .
\end{aligned}
$$

Now, for any $\mu \geqslant 0$, we have $\mu\left\langle\mathcal{O}_{\mu i}^{\dagger} \mathcal{O}_{\mu i}\right\rangle_{\psi} \geqslant 0$, while for any $\mu \leqslant 0$, we have $-\mu\left\langle\mathcal{O}_{\mu i} \mathcal{O}_{\mu i}^{\dagger}\right\rangle_{\psi} \geqslant 0$ for all physically reasonable states $\psi[21]$. Thus,

$$
\begin{aligned}
\left\langle T_{f}\right\rangle_{\psi} & \geqslant-\frac{1}{2 \pi} \int_{0}^{\infty} d \mu \mu S_{\mu}+\frac{1}{2 \pi} \int_{-\infty}^{0} d \mu \mu S_{-\mu} \\
& =-\frac{1}{\pi} \int_{0}^{\infty} d \mu \mu S_{\mu}
\end{aligned}
$$


so we have obtained a state-independent lower bound on $\left\langle T_{f}\right\rangle_{\psi}$. We now proceed to calculate the right-hand side of Eq. (2.23) and show that it is finite. Inserting the definition Eq. (2.16) of $S_{\mu}$ into Eq. (2.23), we find

$$
\left\langle T_{f}\right\rangle_{\psi} \geqslant-\frac{2}{\pi} \int_{0}^{\infty} d \mu \mu \frac{1}{V} \sum_{\boldsymbol{k}}\left|\widehat{g}\left(\omega_{\boldsymbol{k}}+\mu\right)\right|^{2}
$$

Taking the continuum limit

$$
\frac{1}{V} \sum_{\boldsymbol{k}} \longrightarrow \int \frac{d^{3} \boldsymbol{k}}{(2 \pi)^{3}}
$$

the inequality becomes

$$
\begin{aligned}
\left\langle T_{f}\right\rangle & \geqslant-\frac{2}{\pi} \int_{0}^{\infty} d \mu \mu \int_{0}^{\infty} \frac{d^{3} \boldsymbol{k}}{(2 \pi)^{3}}\left|\widehat{g}\left(\omega_{\boldsymbol{k}}+\mu\right)\right|^{2} \\
& =-\frac{2}{\pi} \int_{0}^{\infty} d \mu \mu \frac{1}{8 \pi^{3}} 4 \pi \int_{0}^{\infty} d k k^{2}\left|\widehat{g}\left(\omega_{k}+\mu\right)\right|^{2} \\
& =-\frac{1}{\pi^{3}} \int_{0}^{\infty} d \mu \mu \int_{m}^{\infty} d \omega \omega \sqrt{\omega^{2}-m^{2}}|\widehat{g}(\omega+\mu)|^{2}
\end{aligned}
$$

where we have made a change of variables in the integration to spherical polar coordinates, integrated over the angular variables and changed variable again to the energy $\omega=\sqrt{k^{2}+m^{2}}$. The next step is to make a further change of variable

$$
u=\omega+\mu, \quad v=\omega
$$

after which the quantum inequality Eq. (2.26) becomes

$$
\begin{aligned}
\left\langle T_{f}\right\rangle & \geqslant-\frac{1}{\pi^{3}} \int_{m}^{\infty} d u|\widehat{g}(u)|^{2} \int_{m}^{u} d v v(u-v) \sqrt{v^{2}-m^{2}} \\
& =-\frac{1}{\pi^{3}} \int_{m}^{\infty} d u|\widehat{g}(u)|^{2}\left(\frac{1}{3} u\left(u^{2}-m^{2}\right)^{3 / 2}-\frac{1}{4} u^{4} Q_{3}\left(\frac{u}{m}\right)\right),
\end{aligned}
$$

where the function $Q_{3}(x)$ is given in Eq. (1.2).

Using the translational invariance of the theory to move from a worldline $(t, \mathbf{0})$ to $\left(t, \boldsymbol{x}_{\mathbf{0}}\right)$, we have therefore established the bound

$$
\int d t\left\langle: T_{00}:\right\rangle_{\psi}\left(t, \boldsymbol{x}_{\mathbf{0}}\right) g(t)^{2} \geqslant-\frac{1}{12 \pi^{3}} \int_{m}^{\infty} d u|\widehat{g}(u)|^{2} u^{4} Q_{3}^{D}(u / m)
$$

for any $\boldsymbol{x}_{\mathbf{0}}$, where

$$
Q_{3}^{D}(x)=4\left(1-\frac{1}{x^{2}}\right)^{3 / 2}-3 Q_{3}(x)
$$

as claimed in the Introduction. This bound is clearly finite for any smooth compactly $\operatorname{supported} g$, as $u^{4} Q_{3}^{D}(u / m)$ grows like $u^{4}$ for large $u$, while $|\widehat{g}(u)|^{2}$ decays faster than any inverse polynomial in $u$ as $u \rightarrow \infty$.

\section{MASSLESS CASE}

We now look at the simplest case of the general QWEI derived above; the massless case. We then compare our result for the Dirac field with those for the scalar field derived by Ford and Roman [4, 5] and Fewster and Eveson 8]. Substituting $m=0$ in the first line of Eq. (2.28) and performing the integral over $v$, we immediately find that the four-dimensional QWEI for massless fields takes the form

$$
\left\langle T_{f}\right\rangle_{\psi} \geqslant-\frac{1}{12 \pi^{3}} \int_{0}^{\infty} d u|\widehat{g}(u)|^{2} u^{4}
$$




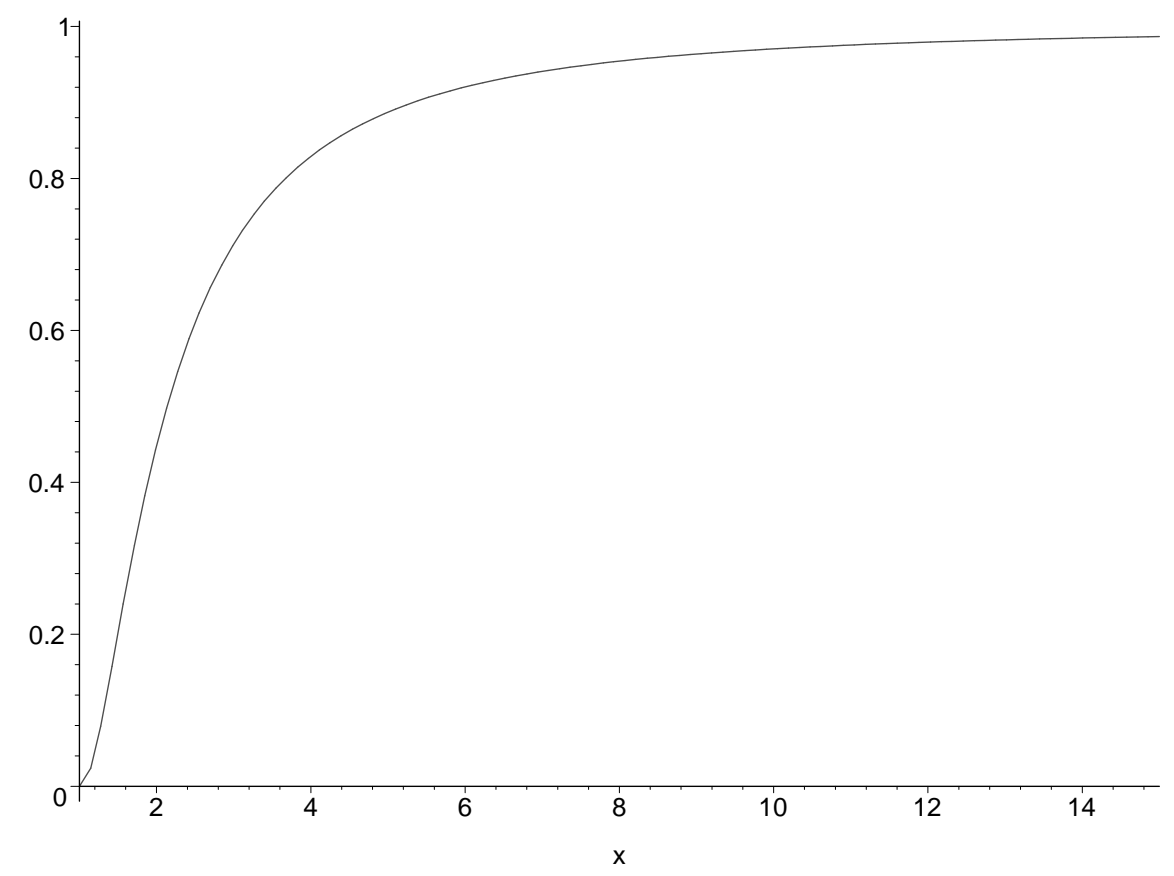

FIG. 1: Plot of $Q_{3}^{D}(x)$.

which is also the $m \rightarrow 0$ limit of Eq. (2.29) because $Q_{3}^{D}(x) \rightarrow 1$ as $x \rightarrow \infty$. Since the integrand is an even function in $u$, we may extend the range of integration to the whole of $\mathbb{R}$ to give

$$
\begin{aligned}
\left\langle T_{f}\right\rangle_{\psi} & \geqslant-\frac{1}{24 \pi^{3}} \int_{-\infty}^{\infty} d u\left|u^{2} \widehat{g}(u)\right|^{2} . \\
& =-\frac{1}{12 \pi^{2}} \int_{-\infty}^{\infty} d t\left|g^{\prime \prime}(t)\right|^{2},
\end{aligned}
$$

where we have used Parseval's theorem to yield a $t$-space version of the quantum inequality in the last line. In passing, we observe that, because $Q_{3}^{D}$ is bounded between 0 and 1 (see Fig. 1), the introduction of a mass only serves to tighten the QWEI bound. Accordingly, Eq. (3.2) also holds for massive Dirac fields.

Comparison with the quantum inequality for the scalar and electromagnetic fields in Minkowski space derived in [8, 11] shows that the massless Dirac bound is weaker than the corresponding scalar bound by a factor of $\frac{4}{3}$, and stronger than the electromagnetic bound by a factor of $\frac{2}{3}$. Since none of these bounds are optimal, it is difficult to draw definite conclusions from this beyond the observation that the bounds are of comparable magnitude.

In particular, for the choice of a Lorentzian sampling function peaked at $t=0$, with characteristic width $\tau$

$$
L(t)=\frac{\tau}{\pi\left(t^{2}+\tau^{2}\right)}
$$

in which $\tau$ sets the averaging timescale, it is straightforward to calculate

$$
\left\langle T_{L}\right\rangle_{\psi} \geqslant-\frac{1}{12 \pi^{2}} \int_{-\infty}^{\infty} d t \frac{\left(2 t^{2}-\tau^{2}\right)^{2} \tau}{\pi\left(t^{2}+\tau^{2}\right)^{5}}=-\frac{36}{2048 \pi^{2} \tau^{4}},
$$

(at least for those states for which the right-hand side is well-defined). This is a slightly stronger result, by a factor of $\frac{3}{16}$, than the quantum inequality derived by Ford and Roman [4, [5] for the scalar field. We have therefore explained why the specific classes of states considered in [17, 18] satisfy the Ford-Roman QWEI. We also observe that a traversable wormhole supported by a Dirac field would be subject to slightly tighter constraints than those envisaged in [2]. 


\section{CONCLUSION}

We have derived a new and explicit QWEI for the Dirac field of mass $m \geqslant 0$ in four-dimensional Minkowski space, by straightforward means. Our argument is related to the general (but inexplicit) QWEI obtained in [16], in that Lemma 1 is at its heart; one may therefore regard it as an improved and streamlined version of [16] (for the particular case of averaging along an inertial trajectory in Minkowski space) with the added benefit of a closed-form expression for the bound. Although it is at present somewhat formal, we expect that our argument can be made fully rigorous (by means of microlocal techniques, cf. [10, 16]) and that it can shed further light on the general situation in curved spacetimes, again leading to explicit bounds.

Our argument is also related to that developed for the scalar field in [8, 9], and which we briefly summarise in Appendix [B] In each case, an identity for Fourier transforms (the convolution theorem or Lemma 1) and the (anti)commutation relations are used to express the averaged energy density as an integral of manifestly positive operators, modulo a $c$-number term which eventually provides the lower bound. Extra care is required in the Dirac case because the classical energy density is unbounded both from above and below.

Finally, in contrast to Vollick's two-dimensional bound [15], our QWEI is not expected to be the sharpest bound possible. Again, it is hoped to address this issue elsewhere.

\section{APPENDIX A: PROOF OF LEMMA 1}

In this appendix, we wish to state and prove the following lemma (originally proved in [16] by a slightly different method): If $f=g^{2}$ for real-valued $g \in C_{0}^{\infty}(\mathbb{R})$, then

$$
\left(\lambda+\lambda^{\prime}\right) \widehat{f}\left(\lambda-\lambda^{\prime}\right)=\int_{-\infty}^{\infty} \frac{d \mu}{\pi} \mu \widehat{g}(\lambda-\mu) \overline{\widehat{g}\left(\lambda^{\prime}-\mu\right)} .
$$

To obtain this result, first notice that the left-hand side is the momentum space integral kernel for the operator

$$
A=p f+f p
$$

where $f$ acts by multiplication and $p=-i d / d x$. That is,

$$
\widehat{A \psi}(\lambda)=\int_{-\infty}^{\infty} \frac{d \lambda^{\prime}}{2 \pi}\left(\lambda+\lambda^{\prime}\right) \widehat{f}\left(\lambda-\lambda^{\prime}\right) \widehat{\psi}\left(\lambda^{\prime}\right)
$$

holds (at least) for all smooth, compactly supported $\psi$. On the other hand, because $f=g^{2}$, we also have

$$
A=p g^{2}+g^{2} p=2 g p g+[p, g] g+g[g, p]=2 g p g
$$

because $[g, p]=i g^{\prime}$, another multiplication operator. Written as an integral operator, we therefore have

$$
\widehat{A \psi}(\lambda)=2 \int_{-\infty}^{\infty} \frac{d \mu}{2 \pi} \widehat{g}(\lambda-\mu) \mu \int_{-\infty}^{\infty} \frac{d \lambda^{\prime}}{2 \pi} \widehat{g}\left(\mu-\lambda^{\prime}\right) \widehat{\psi}\left(\lambda^{\prime}\right) .
$$

Since both (A3) and A5 hold for all smooth compactly supported $\psi$, the two integral kernels must also agree, therefore yielding (A1), using the fact that $\widehat{g}\left(\mu-\lambda^{\prime}\right)=\overline{\widehat{g}\left(\lambda^{\prime}-\mu\right)}$ because $g$ is real.

\section{APPENDIX B: SKETCH OF THE SCALAR FIELD ARGUMENT}

For ease of comparison with our Dirac QWEI, we briefly summarise the scalar field argument of [8, 9]. (Notation differs slightly, and we have specialised to four-dimensional Minkowski space.) This argument begins by observing that the averaged energy density may be written as a sum of terms of the form

$$
\begin{gathered}
S^{ \pm}=\frac{1}{2} \int \frac{d^{3} \boldsymbol{k}}{(2 \pi)^{3}} \frac{d^{3} \boldsymbol{k}^{\prime}}{(2 \pi)^{3}}\left\{\widehat{f}\left(\omega_{\boldsymbol{k}^{\prime}}-\omega_{\boldsymbol{k}}\right) \overline{p(\boldsymbol{k})} p\left(\boldsymbol{k}^{\prime}\right) a^{\dagger}(\boldsymbol{k}) a\left(\boldsymbol{k}^{\prime}\right)\right. \\
\left. \pm \widehat{f}\left(\omega_{\boldsymbol{k}}+\omega_{\boldsymbol{k}^{\prime}}\right) p\left(\boldsymbol{k}^{\prime}\right) p(\boldsymbol{k}) a\left(\boldsymbol{k}^{\prime}\right) a(\boldsymbol{k})+\text { h.c. }\right\}
\end{gathered}
$$


where $a(\boldsymbol{k})$ and $a^{\dagger}(\boldsymbol{k})$ are the annihilation and creation operators of the scalar field, and $p(k)$ is polynomially bounded in $k$. Introducing operators

$$
\mathcal{O}^{ \pm}(\omega)=\int \frac{d^{3} \boldsymbol{k}}{(2 \pi)^{3}}\left\{\overline{\widehat{g}\left(\omega-\omega_{\boldsymbol{k}}\right)} p(\boldsymbol{k}) a(\boldsymbol{k}) \pm \overline{\widehat{g}\left(\omega+\omega_{\lambda}\right) p(\boldsymbol{k})} a^{\dagger}(\boldsymbol{k})\right\},
$$

a calculation using the commutation relations satisfied by the annihilation and creation operators and the convolution theorem in the form

$$
\int_{-\infty}^{\infty} \frac{d \omega^{\prime}}{2 \pi} \widehat{g}\left(\omega-\omega^{\prime}\right) \widehat{g}\left(\omega^{\prime}\right)=\widehat{g^{2}}(\omega)=\widehat{f}(\omega)
$$

(and the fact that $\widehat{g}(u)=\overline{\widehat{g}(-u)}$ as $g$ is real) yields the expression

$$
\int_{0}^{\infty} \frac{d \omega}{2 \pi} \mathcal{O}_{\omega}^{ \pm \dagger} \mathcal{O}_{\omega}^{ \pm}=S^{ \pm}+\int_{0}^{\infty} \frac{d \omega}{2 \pi} \int \frac{d^{3} \boldsymbol{k}}{(2 \pi)^{3}}\left|\widehat{g}\left(\omega+\omega_{\boldsymbol{k}}\right)\right|^{2}|p(\boldsymbol{k})|^{2} .
$$

Since the right-hand side of Eq. (BB4) is (formally) a manifestly positive operator, we conclude that the expectation value of $S^{ \pm}$in any quantum state $\psi$ must satisfy the following bound:

$$
\left\langle S^{ \pm}\right\rangle_{\psi} \geqslant-\int_{0}^{\infty} \frac{d \omega}{2 \pi} \int \frac{d^{3} \boldsymbol{k}}{(2 \pi)^{3}}\left|\widehat{g}\left(\omega+\omega_{\boldsymbol{k}}\right)\right|^{2}|p(\boldsymbol{k})|^{2}
$$

for all (physically reasonable) states $\psi$. It follows that the averaged energy density satisfies a state-independent lower bound. Further manipulation leads to the bound of Eq. (1.1) with $\mathcal{S}=1$.

\section{ACKNOWLEDGMENTS}

The work of CJF was assisted by EPSRC Grant GR/R25019/01 to the University of York.

[1] H. Epstein, V. Glaser, and A. Jaffe, Nuovo Cimento 36, 1016 (1965).

[2] L.H. Ford and T.A. Roman, Phys. Rev. D 53, 5496 (1996).

[3] L.H. Ford, Proc. Roy. Soc. Lond. A364, 227 (1978).

[4] L.H. Ford and T.A. Roman, Phys. Rev. D 51, 4277 (1995).

[5] L.H. Ford and T.A. Roman, Phys. Rev. D 55, 2082 (1997).

[6] É.É. Flanagan, Phys. Rev. D 56, 4922 (1997).

[7] M.J. Pfenning and L.H. Ford, Phys. Rev. D 57, 3489 (1998).

[8] C.J. Fewster and S.P. Eveson, Phys. Rev. D 58, 084010 (1998).

[9] C.J. Fewster and E. Teo, Phys. Rev D 59, 104016 (1999).

[10] C.J. Fewster, Class. Quantum Grav. 17, 1897 (2000).

[11] M.J. Pfenning, Phys. Rev. D 65, 024009 (2002).

[12] C.J. Fewster and M.J. Pfenning, 'A Quantum Weak Energy Inequality for spin-one fields in curved spacetime', arXiv:gr-qc/0303106 to appear J. Math. Phys. (2003).

[13] A.D. Helfer, 'The Hamiltonians of Linear Quantum Fields: II. Classically Positive Hamiltonians', arXiv:hep-th/9908012

[14] É.É. Flanagan, Phys. Rev. D 66, 104007 (2002).

[15] D.N. Vollick, Phys. Rev. D 61, 084022 (2000).

[16] C.J. Fewster and R. Verch, Commun. Math. Phys. 225, 331 (2002).

[17] D.N. Vollick, Phys. Rev. D 57, 3484 (1998).

[18] H. Yu and W. Shu, Phys. Lett. B 570, 123 (2003).

[19] F. Belinfante, Physica (Amsterdam) 6, 887 (1939).

[20] In principle, one could obtain an explicit bound from [16] by estimating various constant quantities arising in the derivation. However, this would require considerable labour and is unlikely to provide a particularly enlightening result.

[21] In a fully rigorous formulation (cf. [16]), one would expect the QWEI to hold for all Hadamard states. 